\title{
Eva KLÍMOVÁ
}

Università di Opava

\section{SUL RAPPORTO TRA LA STRUTTURA DEL PREDICATO E IL GRADO DI DINAMISMO COMUNICATIVO DEL VERBO PREDICATIVO}

\section{INTRODUZIONE}

La struttura del predicato e il grado di dinamismo comunicativo sono concetti che, a prima vista, appartengono a diversi livelli di analisi linguistica: la struttura del predicato è una questione legata allo studio della sintassi, il grado di dinamismo comunicativo è uno dei concetti di base legati alla struttura informativa, ovvero alla prospettiva funzionale dell'enunciato (PFE). Tuttavia, osservare la struttura del predicato proprio in termini di PFE offre non solo un punto di vista diverso da quello puramente sintattico, ma anche una possibile spiegazione delle ragioni per l'uso di un predicato analitico invece di quello sintetico, lì dove una lingua disponga di tutte e due le possibilità. Se da una parte si constata, per l'italiano, una tendenza allo stile nominale, ${ }^{1}$ dall'altra parte bisognerebbe anche pensare a quali sono le conseguenze possibili di questa tendenza, in che modo si manifesta nell'ambito del processo comunicativo. Il fatto che la stessa tendenza si presenti con molta forza in inglese, quindi in due lingue che tipologicamente sono diverse, stimola ulteriormente la nostra curiosità. La nostra intenzione però non è quella di occuparci delle differenze tipologiche tra italiano e inglese. Intendiamo concentrarci su un problema più stretto e ben delineato, cioè sulla distribuzione del grado di dinamismo comunicativo del verbo predicativo, in dipendenza della struttura del predicato.

La struttura del predicato rappresenta una problematica di estrema importanza se si prende in considerazione che il verbo predicativo è indicabile come centro della frase già a tre livelli: A livello sintattico, la funzione centrale del verbo predicativo deriva dal fatto che da questo viene compiuto, per mezzo delle categorie predicative di tempo e di modo, di cui esso è il portatore, l'atto predicativo, ovvero l'atto della costituzione della frase. ${ }^{2}$ A livello semantico, il ruolo centrale del verbo predicativo è legato alle valenze di esso: è il significato del verbo a determinare il numero e il tipo di argomenti e quindi la struttura sintattica della frase. La nostra intenzione è quella di dimostrare la funzione centrale del verbo predicativo anche a un terzo livello: quello della prospettiva funzionale dell'enunciato (PFE). Per poterlo fare riteniamo inevitabile introdurre alcuni concetti di base di cui intendiamo avvalerci in seguito:

1. La teoria della PFE considera l'enunciato come campo comunicativo e gli elementi di esso come diverse unità comunicative.

\footnotetext{
${ }^{1}$ Cfr. Bally (1963 : 415), dove viene constatata per la lingua italiana (e per quella francese) una tendenza a «Lo stile sostantivo » nei costrutti di significato aggettivale (un ponte di pietra, una statua di marmo), nei costrutti di significato avverbiale (con occhio severo, con severità invece di severamente), ma soprattutto nei predicati analitici (prendere parte invece di partecipare).

${ }^{2}$ Cfr. Bauer, Grepl (1975 : 55).
} 
2. Le unità comunicative in questione sono: tema, transizione propria, transizione e rema. ${ }^{3}$ A ciascuna unità comunicativa viene attribuito un certo grado di dinamismo comunicativo (DC).

3. Per grado di DC si intende «la misura relativa in cui un elemento contribuisce allo sviluppo ulteriore della comunicazione». ${ }^{4}$

4. Per tema s'intende quell'elemento dell'enunciato che è portatore del grado di DC più basso, per rema, invece s'intende l'elemento che riporta il grado di DC più alto. ${ }^{5}$

5. Il grado di DC risulta dall'interazione dei quattro fattori della PFE, cioè ordinamento lineare dell'enunciato, struttura semantica dell'enunciato, contesto e, nella lingua parlata, intonazione. ${ }^{6}$ Questi fattori operano in varie lingue con vigore diverso, tuttavia immancabilmente in interazione tra di loro. Se, per esempio, per l'italiano viene constatata una certa libertà nell'ordine delle parole, il grado di DC del costituente rispettivo, oltre che dalla funzione semantica, può essere segnalato dalla posizione nell'enunciato. In altre parole, il costituente che rappresenta un elemento non dipendente dal contesto, può essere confermato nella sua funzione rematica dal suo carattere di «novità» e anche dalla sua posizione finale. Nella lingua inglese la situazione è diversa: il principio guida dell'ordine delle parole si manifesta nella posizione degli elementi secondo le loro funzioni sintattiche. Di conseguenza, il grado di DC dei costituenti dell'enunciato viene indicato dal ruolo semantico ad essi attribuito. Ciò avviene in stretta dipendenza dal coinvolgimento nel contesto.

Vediamo l'esempio: ${ }^{7}$

(1) Io in quella casa i piedi non ce li metterò mai.

GATT p. 184

I'll never set foot in that house again.

LEOP p. 141

Nell'enunciato italiano si osserva l'ordine soggetto - complemento di luogo complemento oggetto - verbo. Il complemento di luogo in quella casa e il complemento oggetto $i$ piedi si osservano in posizione preverbale, sono cioè dislocati in posizione iniziale nella frase. Così, tramite la dislocazione a sinistra, il verbo metterò rimane alla fine della frase, essendo indicato nella funzione rematica per la posizione. In inglese, invece, si osserva l'ordine fisso, cioè soggetto - verbo - complemento oggetto - complemento di luogo. Mentre in

\footnotetext{
${ }^{3}$ Le unità comunicative di tema e di rema sono ulteriormente scomponibili in tema proprio, tema tendente al tema proprio, tema tendente al diatema, diatema, rema e, rema proprio. Svoboda (1991: 432).

${ }^{4}$ Firbas $(1991: 198)$.

${ }^{5}$ Firbas $(1966: 240)$.

${ }^{6}$ Cfr. Firbas (1991: 197).

${ }^{7}$ Gli esempi con l'abbreviazione GATT, sono stati presi dal romanzo Il Gattopardo di Tomasi di Lampedusa e la sua traduzione in inglese con il titolo The Leopard (abbr. LEOP). Gli esempi con l'abbreviazione IND sono stati presi dal romanzo Gli indifferenti di Alberto Moravia e la sua traduzione in inglese con il titolo The Time of Indifference (abbr. TIM), e gli esempi con l'abbreviazione DUB dal libro di racconti di James Joyce Dubliners e la traduzione italiana Gente di Dublino (abbr. GEN).
} 
italiano la struttura informativa dell'enunciato viene segnalata dall'ordine dei costituenti, in inglese è il contesto a indicarla.

\subsection{ESPONENTI DEL VERBO PREDICATIVO}

Per poter osservare il verbo predicativo in riferimento al grado di DC prendendo, allo stesso tempo, in considerazione la struttura del predicato, ci sembra opportuno avvalerci della constatazione di Firbas: «Assessing the communicative function of the verb, we must bear in mind that the verb (finite or non-finite) consists of the notional component and the categorial exponents. It is necessary to distinguish between the information conveyed by the notional component of the verb and the information conveyed by its categorial exponents. For even the information conveyed by the latter participates in the development of the communication.» ${ }^{8}$ Il verbo predicativo, scomponibile in esponenti categoriali $\mathrm{e}$ nella componente nozionale, può partecipare ad alcune unità comunicative:

(2) Vado nelle montagne.

GATT p. 40

La forma verbale vado esprime le categorie grammaticali di persona, di numero, di tempo e di modo. Il segnale della categoria di persona e quello delle categorie di numero rappresentano l'esponente personale e numerale (EPN). Essi, facendo riferimento al soggetto della frase, ricavabile dal contesto e, essendone l'unico indicatore, svolgono la funzione di tema dell'enunciato. (Cfr. con la traduzione in inglese «I' $m$ off to the hills.» [LEOP p. 21], in cui l'EPN della forma verbale am partecipa al tema, assieme al soggetto pronominale $I$.) I segnali della categoria di tempo e di quella di modo sono l'esponente temporale e modale (ETM) e svolgono la funzione di transizione propria (d'ora in poi solo $\mathrm{TR}_{\text {prop}}$ ). La funzione dell'ETM come transizione propria è immutabile, perché esso è obbligatoriamente presente e riporta informazione sull'inserimento temporale e modale della frase. ${ }^{9}$ Fungendo da transizione propria, l'ETM rappresenta «a centre within the sentence/clause/semiclause viewed as a distributional field of $C D . \gg{ }^{10} \mathrm{La}$ componente nozionale del verbo svolge la funzione di transizione perché, da una parte, rappresentando un elemento non ricavabile dal contesto, porta un grado di DC più alto rispetto all'ETM, dall'altra parte, però, porta un grado di DC più basso rispetto all'avverbiale nelle montagne/to the hills che funge da rema. L'esempio (2), dal punto di vista delle unità comunicative, può essere delineato come segue:

$$
\begin{array}{cc}
\text { Vado } & \text { nelle montagne. } \\
\downarrow & \downarrow \\
{[\text { TEMA (EPN) }} & \rightarrow \mathrm{TR}_{\text {prop }}(\text { ETM }) \rightarrow \text { TRANSIZIONE] } \rightarrow[\text { REMA] }
\end{array}
$$

\footnotetext{
${ }^{8}$ Firbas $(1992: 70)$.

${ }^{9}$ Cfr. Svoboda (1989: 66).

${ }^{10}$ Firbas $(1992: 89)$.
} 


\subsection{LA FUNZIONE DELLA COMPONENTE NOZIONALE DEL VERBO PREDICATIVO}

La componente nozionale del verbo pieno del predicato sintetico, cioè verbale, svolge di solito la funzione di transizione, non di rado però anche la funzione di rema. ${ }^{11}$ Per rendere la spiegazione più semplice possibile, diciamo che lì dove nell'enunciato è presente un elemento che, per quanto riguarda il grado di DC, supera la componente nozionale del verbo, quest'ultima funge da transizione. Invece lì dove nell'enunciato un elemento del genere non è presente, la componente nozionale del verbo è rema. Vediamo gli esempi con un predicato sintetico in tutte e due le lingue:

(3) ... e Michele guardava Leo.

IND p. 26

... and Michele was looking at Leo.

TIM p. 23

(4) Tutti ci guardavano.

IND p. 6

Everyone was looking at us.

TIM p. 6

Nel caso dell'esempio (3), la componente nozionale del verbo guardare/look at funge da transizione perché il complemento oggetto Leo, assumendo la funzione semantica di specificazione, ${ }^{12}$ la supera per il grado di DC e funge da rema. La situazione è diversa nell'esempio (4): il complemento pronominale ci/us rappresenta un elemento recuperabile dal contesto e quindi fa parte del tema. Non essendoci nessun altro elemento che superi per il grado DC la componente nozionale del verbo, quest'ultima funge da rema.

Prima di delineare la funzione della componente nozionale del verbo nei predicati analitici bisogna precisare che cosa si intende, in quest'articolo, per predicato analitico: fanno parte di un predicato analitico i verbi copulativi che sono privi di significato lessicale, e verbi che, pur svolgendo la funzione di copula, mantengono qualche significato lessicale. Vediamo l'esempio:

(5) a. $\grave{E}$ pallido.

b. $\dot{E}$ diventato pallido.

Nella variante (5a) il verbo essere funge da copula e l'aggettivo pallido esprime lo stato. La componente nozionale del verbo copulativo è pari allo zero e quindi il

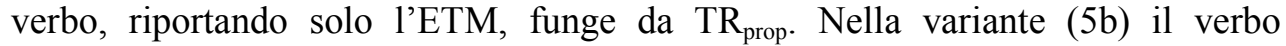
diventare, oltre a fungere da copula, riporta il significato di «passaggio da una condizione all'altra», cioè il significato del cambiamento. La componente nozionale del verbo supera, per quanto riguarda il grado di DC, la copula della variante (5a) e tende a far parte di transizione. L'aggettivo pallido esprime il risultato del cambiamento.

Nella nostra analisi verranno prese in considerazione e classificate alcune strutture come predicati analitici verbo-nominali che, per la forma, si oppongono ai predicati verbali. Sono classificabili come predicati analitici strutture con un verbo che, svuotatosi dal significato lessicale, decade per fungere da verbo categoriale, cioè verbo che riporta le categorie verbali. Nell'esempio successivo

\footnotetext{
${ }^{11}$ Cfr. Svoboda (1989: 65), Firbas (1992: 70-71).

${ }^{12}$ Per le funzioni semantiche degli elementi dell'enunciato cfr. Klímová (2004). 
viene messo a confronto un enunciato italiano con un predicato sintetico a cui corrisponde, nell'enunciato inglese, un predicato analitico:

(6) Ma anche si burlava di lei. IND p. 19

But was making a fool of her into the bargain. TIM p. 17

Con la forma verbale si burlava del predicato sintetico vengono coperte due unità comunicative: quella di $\mathrm{TR}_{\text {prop }}$ ('ETM) e quella di rema (la componente nozionale). Nella versione inglese, invece, si osserva un predicato analitico make a fool (corrispondente alla locuzione verbale italiana «farsi beffe di qualcuno»o «prendersi gioco di qualcuno»). Il verbo inglese make, svuotatosi quasi completamente dal significato lessicale, funge da verbo categoriale: l'ETM della forma was making funge da $\mathrm{TR}_{\text {prop, }}$ la sua componente nozionale è osservabile nel passaggio da $\mathrm{TR}_{\text {prop }}$ a transizione. A svolgere la funzione di rema è il sostantivo fool. Nella differenza tra il verbo italiano pieno e il costrutto analitico inglese si manifesta la tendenza alla sinteticità dell'italiano nel confronto con la tendenza allo stile non-verbale in inglese. ${ }^{13}$

Il rapporto tra la struttura del predicato e il grado di DC del verbo predicativo sarà successivamente osservato in diversi tipi di predicati. Man mano con il rafforzamento del significato lessicale del verbo predicativo, è registrabile l'aumento del grado di DC della componente nozionale e il suo spostamento dall'unità di $\mathrm{TR}_{\text {prop }}$ a quella di transizione, o a quella di rema.

\section{LA COMPONENTE NOZIONALE DEI PREDICATI ANALITICI}

Visto che, dal punto di vista delle unità comunicative, il verbo dei predicati analitici può fungere «solo» da transizione, la nostra attenzione si concentrerà soprattutto su questa unità, nell'ambito della quale si può distinguere un centro, una periferia inferiore e una superiore. Nell'analisi che segue, il centro della transizione sarà indicato come transizione ${ }^{0}$, la periferia inferiore come transizione ${ }^{-}$ e la periferia superiore come transizione ${ }^{+} .{ }^{14}$

La misura di sinteticità $\mathrm{o}$, al contrario, quella di analiticità di una lingua si manifesta anche nell'atto di formazione delle parole: nel processo di derivazione, con un prefisso e un suffisso, viene creato un verbo che, rispetto alla struttura del predicato, risulta essere predicato sintetico. Potrebbe apparire questo come un fatto di poca rilevanza. La assume nel momento in cui si considera che le capacità di derivazione di una lingua si riflettono anche nelle possibilità di modificare le caratteristiche aspettuali dell'azione verbale. ${ }^{15}$

L'esempio successivo deve evidenziare l'impatto che la struttura del predicato esercita sulla distribuzione del DC nelle unità comunicative dell'enunciato:

(7) Impallidì,... She turned pale, and ...
IND p. 26

TIM p. 23

In italiano, nel processo di doppia derivazione, con il prefisso im- e il suffisso -ire, è stato creato dall'aggettivo pallido il verbo incoativo impallidire che esprime due

\footnotetext{
${ }^{13}$ Cfr. Firbas (1959 : 51).

${ }^{14}$ Cfr. Svoboda (1989 : 82).

${ }^{15}$ Cfr. Hamplová (1992 : 85).
} 
significati: quello del cambiamento, e quello del risultato del cambiamento. Sintatticamente, il verbo italiano impallidì rappresenta un predicato sintetico. Nel costrutto inglese she turned pale i due significati sono separati l'uno dall'altro in due elementi del predicato analitico: Il verbo turn esprime il significato del cambiamento, mentre l'aggettivo pale il significato del risultato del cambiamento. (Cfr. diventare pallido dell'esempio (5b.) sopra.)

La differenza sintattica tra il predicato sintetico e quello analitico si riflette sulla funzione della componente nozionale del verbo predicativo: la forma verbale impallidi, oltre a riportare l'EPN di tema e l'ETM di $\mathrm{TR}_{\text {prop}}$, comprende la componente nozionale che funge da rema. La forma verbale inglese she turned, dopo la separazione dell'ETM, contiene la componente nozionale corrispondente al significato diventare. Essa funge da transizione ${ }^{-}$essendo superata, per quanto riguarda il grado di DC, dall'aggettivo pale che funge da rema (l'EPN è riportato dal soggetto pronominale she).

Prima di concentrarci su diversi tipi di predicati, riportiamo alcuni esempi in cui la componente nozionale del verbo predicativo è registrabile in una scala, a partire dai verbi che sono privi di significato lessicale, fino ai verbi classificabili come verbi pieni. Con il rafforzamento del significato lessicale del verbo, aumenta il grado di DC della componente nozionale:

(8) Perché tutto questo mi è indifferente.

IND p. 13

Because all this is quite indifferent to me.

TIM p. 12

In tutte e due le versioni si osserva il predicato verbo-nominale composto dalla copula essere/be e un aggettivo. Il verbo copulativo, privo di significato lessicale, riporta solo l'ETM e funge da $\mathrm{TR}_{\text {prop. }}$ È l'aggettivo indifferente/indifferent a fungere da rema. Il significato lessicale del verbo predicativo nell'esempio successivo è più forte:

(9) ... lui ... restava indifferente.

IND p. 33

... he ... remained indifferent.

TIM p. 29

In tutte e due le versioni è il verbo restare/remain a far parte del predicato analitico. Si distingue dal verbo copulativo dell'esempio precedente nel senso che esprime la permanenza dello stato di indifferenza, esprime cioè una fase dell'azione. Torneremo ai verbi fraseologici più avanti. In italiano, l'aspetto durativo dell'azione, oltre a essere espresso dal verbo rimanere, risulta dalla forma dell'imperfetto. Con l'indicazione della fase dell'azione, la componente nozionale supera il limite di $\mathrm{TR}_{\text {prop }}$ ed è classificabile come transizione'. Va aggiunto che è l'aggettivo a fungere da rema.

Finora tra, la versione italiana e quella inglese non è stata registrata nessuna differenza rilevante. Gli esempi successivi sono riportati per dimostrare che in tutte e due le lingue il predicato analitico può essere sostituito da un predicato sintetico. Ciò naturalmente si riflette nelle unità comunicative di $\mathrm{TR}_{\text {prop }} \mathrm{e}$ di transizione:

Tutto mi è indifferente ...

IND p. 34

Nothing matters in the least to me.

TIM p. 31 
(11) Quanto tutto questo m'importi poco, non ve lo potete ... IND p. 35 How utterly indifferent I am to it all. You can't ...

TIM p. 31

Nella versione italiana dell'esempio (10) e dell'esempio (11) si alternano un predicato analitico con un predicato sintetico. Nella versione inglese, viceversa. Tuttavia, la distribuzione del grado di DC rimane come è stato descritto sopra: il verbo copulativo essere/be riporta l'ETM e funge da $\mathrm{TR}_{\text {prop. }}$. A fungere da rema è l'aggettivo indifferente/indifferent. Nel caso del predicato sintetico, i verbi matter e importare coprono due unità comunicative: l'ETM quella di $\mathrm{TR}_{\text {prop}}$, la componente nozionale del verbo quella di rema.

Come passo successivo, il verbo sarà osservato prima nell'ambito dei predicati verbo-nominali che presentano $\mathrm{i}$ verbi copulativi essere/be, avere/have $\mathrm{e}$ parere/seem; in seguito ci si occuperà del verbo nell'ambito dei predicati che comprendono i verbi fraseologici cominciare, continuare, smettere/begin, go on, continue, stop, oppure $\mathrm{i}$ verbi che esprimono il cambiamento di stato diventare/become. Successivamente si esamineranno alcuni esempi con i verbi fare/make e dare/give nei predicati analitici che perdono, parzialmente o del tutto, il significato lessicale e fungono da verbo categoriale. Alla fine verranno riportati gli esempi con predicati sintetici. Con il passaggio dal predicato analitico a quello sintetico, si registra un rafforzamento della componente nozionale del verbo predicativo, che, separandosi dall'ETM di $\mathrm{TR}_{\text {prop }}$, passa verso l'unità comunicativa di transizione, ovvero verso il rema.

\subsection{VERBI COPULATIVI}

\subsubsection{Verbi copulativi essere e avere}

Negli esempi successivi si osservano i predicati con i verbi essere e avere. Essi, avendo perso il significato lessicale, fungono da copula:

$$
\begin{aligned}
& \text {... e che sono contentissimo. IND p. } 19 \\
& \text {... and that I am extremely happy. TIM p. } 17 \\
& \text { (13) In fondo } h a \text { ragione. IND p. } 18 \\
& \text { Really and truly, he's right. } \quad \text { TIM p. } 15
\end{aligned}
$$

Nell'esempio (12) si osserva lo stesso verbo copulativo, cioè essere/be, e nell'esempio (13) al verbo italiano avere corrisponde in inglese il verbo be. Le forme verbali non fanno altro che riportare l'EPN tematico e l'ETM di $\mathrm{TR}_{\text {prop. }}$. Sono gli aggettivi contentissimo/(extremely) happy nell'esempio (12) e il sostantivo ragione e l'aggettivo right nell'esempio (13) a fungere da rema.

\subsubsection{Cambiamento di stato}

In italiano per riportare il significato del cambiamento, vengono adoperati tanto gli strumenti analitici, quanto quelli sintetici. Per strumento analitico si intende un predicato composto contemporaneamente da un verbo che esprime il cambiamento di stato e da un elemento di carattere nominale, che esprime il 
risultato del cambiamento. Per strumento sintetico si intende un verbo derivato da un aggettivo. ${ }^{16}$ Vediamo gli esempi:

La scena diventava sentimentale.

IND p. 22

The scene was becoming sentimental.

TIM p. 20

Nel predicato analitico il verbo copulativo diventare esprime il cambiamento e l'aggettivo sentimentale il risultato del cambiamento. ${ }^{17}$ Rispetto ai verbi copulativi essere e avere, la componente nozionale è più forte ed è registrabile nella periferia dell'unità comunicativa di transizione: funge cioè da transizione-' L'aggettivo è rema. (Cfr. la distribuzione di DC nella traduzione inglese: La componente nozionale del verbo become è transizione-, l'aggettivo sentimental è rema.) Nell'esempio successivo la struttura del predicato è diversa, il che si riflette nella distribuzione di DC:

(15) (Era tardi) quando mi addormentai. (It was late) when I fell asleep.
GEN p. 2

DUB $p .3$

Il verbo addormentarsi del predicato sintetico esprime il significato del cambiamento (a differenza del verbo dormire che esprime lo stato). Questo significato rappresenta la transizione-, la componente nozionale è rema. Al predicato sintetico italiano corrisponde, nella versione inglese dell'esempio, un predicato analitico. Il verbo fall riporta il significato del cambiamento e funge da transizione'. È l'aggettivo asleep a fungere da rema. Quindi si può constatare che il grado di DC della componente nozionale del verbo predicativo italiano è più alto rispetto a quello della componente nozionale del verbo inglese. Anche nell'esempio successivo, al predicato sintetico italiano corrisponde un predicato analitico inglese:

$$
\begin{array}{ll}
\text { Perché vi arrabbiate per delle cose che ... } & \text { IND p. } 19 \\
\text { Because you get into a rage over things that ... } & \text { TIM p. } 17
\end{array}
$$

Il verbo arrabbiarsi, derivato con il prefisso $a$ - e il suffisso -are dal sostantivo rabbia, rappresenta un predicato sintetico. Di nuovo, il significato del cambiamento è transizione ${ }^{-}$, la componente nozionale è transizione ${ }^{0}$. Nel predicato analitico inglese get into a rage il verbo get esprime il cambiamento e funge da transizione-. È la struttura preposizionale into a rage a fungere da transizione ${ }^{0}$. A svolgere la funzione di rema è la specificazione per delle cose/over things. Anche in questo caso il grado di DC della componente nozionale del verbo italiano è più alto rispetto a quello della componente nozionale del verbo inglese.

Gli esempi riportati in questa sezione dimostrano le possibilità dell'italiano di esprimere il significato del cambiamento in due modi diversi: esplicitamente con il verbo diventare del predicato verbo-nominale, o implicitamente con un verbo prefissato del predicato verbale. In inglese, il significato del cambiamento viene espresso con diversi verbi copulativi nei predicati analitici. Questa tendenza

\footnotetext{
${ }^{16}$ Qualche volta, oltre a riportare il significato del cambiamento, il verbo esprime anche una fase dell'azione. Cfr. Hamplová (1992 : 124).

${ }^{17} \mathrm{La}$ forma dell'imperfetto del verbo diventare esprime il cambiamento nel suo proseguimento e funge da strumento implicito d'indicazione di questa fase d'azione. (Cfr. più avanti la fase dell'azione.)
} 
all'uso dei predicati analitici in inglese dimostra la misura di analiticità, a livello sintattico, superiore rispetto all'italiano.

\subsubsection{Impressione soggettiva}

Oltre ai verbi essere/be e avere/have, e oltre ai verbi come diventare/become che esprimono il cambiamento di stato, fungono da verbi copulativi anche quelli che hanno il significato di impressione soggettiva, come per es. parere, sembrare in italiano, e look, seem in inglese:

Era del tutto rassegnato.

GEN p. 32

He was quite resigned.

DUB p. 7

(18) La testa pareva ancor più piccola su quelle spalle piene ...

IND p. 31

Her head looked even smaller above those full shoulders ...

TIM p. 28

Nell'esempio (17) si osserva, in tutte e due le versioni, il verbo copulativo essere/be, privo di qualsiasi significato lessicale, in funzione di $\mathrm{TR}_{\text {prop. }} \mathrm{L}$ 'aggettivo rassegnato/resigned è rema. I verbi parere/look nell'esempio (18), esprimendo impressione soggettiva, hanno, rispetto ai verbi essere/be, un significato lessicale. Questa differenza fa sì che la loro componente nozionale sia osservabile come transizione ${ }^{-18}$ In altre parole, il grado di DC dei verbi con il significato di impressione soggettiva è più alto rispetto al grado di $\mathrm{DC}$ dei verbi copulativi privi di significato lessicale.

\subsection{DAL PREDICATO ANALITICO AL PREDICATO SINTETICO}

\subsubsection{Il predicato analitico con i verbi fare e dare}

In questa sezione viene osservata la componente nozionale dei verbi fare e dare che, svuotandosi dal loro significato lessicale, fanno parte dei predicati analitici:
Fai del moto.
GEN p. 28
Take exercise.
DUB p. 2

Al verbo predicativo fare corrisponde nella versione inglese il verbo take. Tutti e due non fanno altro che riportare le categorie verbali: il loro EPN è tema dell'enunciato, l'ETM è $\mathrm{TR}_{\text {prop }}$ (cfr. la possibilità dell'italiano di sostituire il costrutto analitico fare moto con il predicato sintetico muoversi). È il sostantivo moto/exercise a fungere da rema.

Nell'esempio successivo il predicato analitico nella versione italiana, corrisponde al predicato sintetico nella versione inglese:

$$
\begin{array}{ll}
\ldots \text { e anzi quasi mi fa piacere. } & \text { IND p. } 13 \\
\text { In fact, it almost pleases me. } & \text { TIM p. } 12
\end{array}
$$

In questo caso il verbo fare non può essere considerato come verbo privo di alcun significato lessicale. Anzi, essendo sostituibile con un verbo pieno, come

${ }^{18}$ Cfr. Svoboda (1989 : 80). 
«provocare, suscitare», è scomponibile in ETM e in componente nozionale. Il primo rappresenta la $\mathrm{TR}_{\text {prop, }}$ la seconda funge da transizione- Di nuovo è il sostantivo a svolgere la funzione di rema. Il verbo predicativo inglese please include tutte e due le unità comunicative: l'ETM quella di $\mathrm{TR}_{\text {prop}}$, la componente nozionale quella di rema.

Anche nell'esempio seguente il verbo fare viene osservato in un predicato analitico, questa volta, però, in funzione causativa:

..., che mi aveva fatto imparare a memoria.

... which he had made me learn by heart.
GEN p. 30

DUB p. 5

In questa funzione, al verbo fare corrisponde il verbo inglese make. A tutti e due i verbi è attribuibile il significato «indurre (qualcuno) a fare (qualche cosa)». Anche in questo caso la componente nozionale del verbo predicativo rappresenta la transizione-. A fungere da rema è la struttura con l'infinito imparare a memoria/learn by heart.

Nell'esempio successivo è il verbo dare a partecipare a un predicato analitico:
Nannie dette l'esempio e ...
GEN p. 31
Nannie gave the lead and ...
DUB p. 6

I verbi dare/give, semanticamente parzialmente svuotati, riportano l'ETM di $\mathrm{TR}_{\text {prop. }}$. La componente nozionale funge da transizione, il sostantivo esempio/lead da rema.

Nell'ultimo esempio di questa sezione i verbi fare e make sono verbi pieni:
... chi te lo ha fatto?
IND p. 13
Who made it for you?
TIM p. 13

Il verbo fare/make di quest'esempio è un verbo transitivo accompagnato da un complemento oggetto pronominale lo/it. Visto che il complemento oggetto pronominale rappresenta un elemento tematico, è la componente nozionale del verbo a fungere da rema.

\subsubsection{Fase dell'azione}

Per fase dell'azione si intende l'inizio, il proseguimento e la fine di essa. La fase dell'azione viene indicata esplicitamente con un verbo fraseologico che assieme all'infinito del verbo pieno, costituiscono un predicato analitico. Implicitamente, la fase dell'azione può risultare dal significato lessicale del verbo del predicato sintetico, ${ }^{19}$ in italiano però può essere segnalata anche dagli strumenti morfologici. Nel primo caso, la fase dell'azione sarebbe considerata come categoria semantico-lessicale, nel secondo caso essa è legata all'aspetto dell'azione, ed è in stretto rapporto con la temporalità della frase. ${ }^{20}$ In ogni caso, dalla struttura del predicato deriva la distribuzione di DC nell'ambito delle unità comunicative di $\mathrm{TR}_{\text {prop }}$ e di transizione. Siamo del parere che lì dove la fase dell'azione viene indicata esplicitamente da un verbo fraseologico, essa è individuabile come un componente di transizione. Invece lì dove la fase deriva

\footnotetext{
${ }^{19}$ Cfr. Daneš, Hlavsa, Grepl (1987 : 24).

${ }^{20}$ Cfr. Hamplová (1992: 8).
} 
implicitamente dal significato lessicale del verbo del predicato sintetico o dall'aspetto, essa rimane vicino a $\mathrm{TR}_{\text {prop. }}{ }^{21} \mathrm{E}$ l'esempio successivo a evidenziare il nostro approccio:

(24) Il fumo azzurro sali sottile dalla ...

IND p. 23

Blue smoke began to ascend in thin spirals from ... $\quad$ TIM p. 21

Il predicato sintetico italiano salì corrisponde al predicato analitico inglese began to ascend. L'ETM del verbo italiano è $\mathrm{TR}_{\text {prop. }}$. Con la forma del perfetto semplice viene riportato l'aspetto incoativo, ${ }^{22}$ che è osservabile come un altro componente tra $\mathrm{TR}_{\text {prop }}$ e transizione, ed è quindi considerabile o come $\mathrm{TR}_{\text {prop }}{ }^{+}$, oppure come transizione'. La componente nozionale è transizione ${ }^{0}$. L'ETM della forma verbale inglese began è $\mathrm{TR}_{\text {prop }}$, la sua componente nozionale è transizione'. A fungere da transizione ${ }^{0}$ è l'infinito ascend. Dall'analisi risulta che la componente nozionale del predicato sintetico italiano supera, per quanto riguarda il grado di DC, il verbo del predicato analitico inglese.

Successivamente verranno presi in considerazione tanto gli strumenti lessicali, quanto quelli grammaticali, funzionali all'indicazione dell'inizio, del proseguimento e della fine dell'azione, riguardo alla struttura delle unità comunicative dell'enunciato.

\subsubsection{L'inizio dell'azione}

Nel primo esempio, la fase iniziale dell'azione, in tutte e due le versioni, è indicata in modo esplicito, cioè con un verbo fraseologico:

... Salina che per la terza volta ricominciò a piangere; GATT p. 183

... Salina, who began weeping for the third time; LEOP p. 140

Il predicato analitico italiano ricominciò a piangere corrisponde a quello inglese began weeping. Quindi la distribuzione del DC è uguale in tutte e due le lingue: La forma verbale ricominciò/began, oltre a riportare l'ETM, esprime l'inizio dell'azione e funge da transizione'. L'infinito piangere e il participio weeping sono rema. Nell'esempio successivo si osserva, nella versione italiana, un predicato sintetico:

Poi la lampada si accese.

Then the lamp went on.

IND p. 26

TIM p. 23

Il verbo italiano accendersi, nel significato di cominciare a funzionare, esprime l'inizio dell'azione e anche l'azione stessa. Contemporaneamente, l'aspetto incoativo è indicato dalla forma del perfetto semplice. Al verbo italiano accendersi corrisponde il costrutto inglese go on, con il significato «begin to operate». Una sola forma verbale esprime l'inizio dell'azione e la componente nozionale. L'aspetto incoativo rappresenta la $\mathrm{TR}_{\text {prop }}{ }^{+}$, la componente nozionale è rema.

\footnotetext{
${ }^{21}$ Cfr. Svoboda (1989: 74).

${ }^{22}$ Sulla capacità del perfetto semplice di esprimere l'aspetto ingressivo cfr. per es. Hamplová (1992 : 90).
} 


\subsubsection{Il proseguimento dell'azione}

Anche il proseguimento dell'azione può essere indicato sia esplicitamente, sia implicitamente. Nel primo esempio, esso viene indicato esplicitamente, cioè con un verbo fraseologico nel predicato analitico:
... così continuai a mangiare come se ...
GEN p. 28
... so I continued eating as if ...
DUB p. 2

Il significato riportato dai verbi fraseologici continuare/continue rappresenta la transizione'. A fungere da rema è l'infinito italiano mangiare e il gerundio inglese eating. Nell'esempio successivo si osserva un predicato sintetico, e cioè il passaggio dalla indicazione esplicita, a quella implicita del proseguimento dell'azione:
Tirava la gonna, ...
IND p. 7
He kept pulling at her skirt ...
TIM p. 7

La forma dell'imperfetto tirava, oltre all'incompiutezza, indica l'azione nel suo proseguimento. Così, con uno strumento morfologico, cioè in sintesi di una forma verbale, si realizzano l'ETM di $\mathrm{TR}_{\text {prop}}$, il significato della fase dell'azione di $\mathrm{TR}_{\text {prop }}{ }^{+} \mathrm{e}$ la componente nozionale di transizione ${ }^{0}$. Mentre in italiano la fase di proseguimento viene indicata implicitamente, il costrutto analitico inglese con il verbo fraseologico keep la indica esplicitamente: l'ETM è $\mathrm{TR}_{\text {prop }}$, il significato di proseguimento è transizione-. È il gerundio pulling a fungere da transizione ${ }^{0}$. Il grado di DC della forma verbale italiana tirava è superiore rispetto a quella del verbo inglese kept.

\subsubsection{La fine dell'azione}

Gli strumenti lessicali che servono per l'indicazione esplicita della fase finale dell'azione sono i verbi fraseologici come smettere/stop nei costrutti dei predicati analitici, accompagnati da un verbo pieno:

(29) Torino non vuole cessare di essere capitale.

GATT p. 209

Turin doesn't want to cease being a capital. $\quad$ LEOP p. 161

Il verbo cessare/cease esprime la fine dell'azione e funge da transizione-. La componente nozionale dell'infinito italiano essere e del gerundio inglese being è transizione ${ }^{0}$. È il nome predicativo capitale/capital a svolgere la funzione di rema. Nell'esempio successivo il verbo cessare è adoperato come verbo pieno nel predicato sintetico, il che, naturalmente, si riflette nella distribuzione di DC:

(... Salina che per la terza volta ricominciò a piangere;) a poco a poco $\mathrm{i}$ singhiozzi però decrebbero, cessarono.

GATT p. 183

(... Salina, who began weeping for the third time;) gradually her sobs lessened and then stopped. $\quad$ LEOP p. 140

Con l'EPN la forma verbale cessarono/stopped partecipa, assieme al soggetto singhiozzi/sobs al tema. L'ETM è $\mathrm{TR}_{\text {prop }}$, la componente nozionale è rema.

Gli esempi dimostrano che lo strumento di base per l'indicazione della fase dell'azione sono, in tutte e due le lingue, i verbi fraseologici, e che questi, almeno 
per quanto riguarda l'italiano, «entrano in concorrenza» con strumenti lessicali, cioè con i verbi derivati, e con quelli morfologici, cioè la forma verbale. Dall'analisi degli esempi risulta chiaro che la frontiera tra l'unità comunicativa di $\mathrm{TR}_{\text {prop }}$ e quella di transizione può essere poco distinguibile. Siamo del parere che l'indicazione esplicita della fase dell'azione in un predicato analitico è considerabile come transizione, e l'indicazione implicita che risulta dalla forma del verbo è considerabile come componente in periferia superiore di $\mathrm{TR}_{\mathrm{prop}}$.

\subsection{LA COMPONENTE NOZIONALE DEI PREDICATI SINTETICI}

Gli esempi di questa sezione presentano predicati sintetici. Tuttavia, il modo in cui i componenti semantici del verbo si realizzano nell'ambito delle unità comunicative, non è uguale. Prendiamo come punto di partenza il caso in cui le due versioni non si differenziano:
Tornò alla sua pipa e ...
GEN p. 28
He returned to his pipe and ...
DUB p. 2
... e ce li passò.
GEN p. 31
... passed them to us.
DUB p. 7

La componente nozionale del verbo tornare/turn nell'esempio (31) è transizione, il complemento indiretto alla sua pipa/to his pipe è rema. Nel caso dell'esempio (32), la componente nozionale del verbo passare/pass funge da rema perché i complementi pronominali ce li/them to us rappresentano elementi recuperabili dal contesto e, assieme all'EPN, appartengono a tema. La situazione non è così semplice negli esempi che seguono:

... quando scesi per cena.

GEN p. 27

... when I came downstairs to supper.

DUB p. 1

Il verbo italiano scendere esprime «movimento giù». Il verbo comprende due componenti semantici: Il primo, movimento, è transizione ${ }^{0}$, il secondo, giù, è considerabile come transizione ${ }^{+}$. Il complemento per cena funge da rema. Nel costrutto inglese come downstairs il verbo come esprime movimento, mentre è l'avverbiale downstairs a esprimere la direzione del movimento. La componente nozionale del verbo è transizione ${ }^{0}$, l'avverbio corrisponde a transizione ${ }^{+}$. Il grado di DC della componente nozionale del verbo italiano è più alto rispetto a quello della componente nozionale del verbo inglese.

Nell'esempio successivo il predicato sintetico viene osservato solo in italiano:

$$
\begin{array}{ll}
\text { ('Ma sì!') Leo ..., l'afferrò per la gonna. } & \text { IND p. } 7 \\
\text { ('Ah, do!') Leo ... and took hold of her skirt again. } & \text { TIM p. } 7
\end{array}
$$

Il verbo afferrare, derivato dal sostantivo ferro, significa «prendere velocemente e con forza». Quindi anche se i verbi afferrare e prendere sono considerabili sinonimi, il significato del primo è più ricco rispetto a quello del secondo. La stessa differenza si può registrare in inglese tra il significato del verbo take e il costrutto take hold (of). Mentre il verbo italiano afferrare riporta due componenti semantici, quello di prendere e quello di modo di prendere, nella struttura analitica inglese take hold (of) questi due componenti semantici vengono separati uno dall'altro: il primo viene riportato dal verbo take, il secondo dal sostantivo hold. 
Questa differenza si riflette nella struttura dell'unità comunicativa di transizione. Il significato prendere rappresenta la transizione ${ }^{0}$, il significato «velocemente e con forza» è considerabile transizione ${ }^{+}$. Nel costrutto inglese, la componente nozionale del verbo take è transizione ${ }^{0}$. A fungere da transizione ${ }^{+}$è il sostantivo hold. Quindi si può costatare che il grado di DC della componente nozionale del verbo italiano supera quello della componente nozionale del verbo inglese.

La situazione è diversa nell'ultimo esempio: in tutte e due le versioni è un predicato sintetico, tuttavia il verbo italiano è, per quanto riguarda il significato, più debole:

Era stato deposto nella bara.

He had been coffined.

GEN p. 31

DUB p. 6

Il verbo predicativo deporre è accompagnato dal complemento di luogo nella bara. La componente nozionale è transizione ${ }^{0}$, il complemento di luogo è rema. In inglese si osserva il verbo coffin che ha origine dalla conversione del sostantivo coffin. È la componente nozionale a fungere da rema. In questo caso, il grado di DC della componente nozionale del verbo inglese supera quello della componente nozionale del verbo italiano.

\section{CONCLUSIONE}

Il rapporto tra la struttura del predicato e il grado di dinamismo comunicativo (DC) del verbo predicativo è stato osservato in diversi tipi di predicati: Si è partito dai predicati analitici con i verbi copulativi essere/be e avere/have, la cui componente nozionale è pari allo zero, passando ai predicati che contengono un verbo che esprime il cambiamento di stato, un verbo con il significato «impressione soggettiva», o un verbo fraseologico, per finire con i predicati sintetici contenenti un verbo pieno. Gradualmente, con il rafforzamento del significato lessicale del verbo predicativo, cambia la funzione della componente nozionale di esso riguardo alle unità comunicative dell'enunciato: un verbo copulativo, semanticamente vuoto, riporta l'ETM e funge da $\mathrm{TR}_{\text {prop}}$; la componente nozionale dei verbi categoriali si realizza nell'ambito di transizione e la componente nozionale del verbo pieno funge da transizione, o da rema. Schematicamente, la funzione della componente nozionale del verbo predicativo viene registrata nella tabella seguente:

\begin{tabular}{|c|c|c|}
\hline $\begin{array}{l}\text { Unità } \\
\text { comunicativa }\end{array}$ & Italiano & Inglese \\
\hline $\mathrm{TR}_{\text {prop }}{ }^{0}$ & $\begin{array}{l}\text { ETM, componente nozionale } \\
\text { «0» dei verbi copulativi } \\
\text { essere, avere }\end{array}$ & $\begin{array}{l}\text { ETM, componente nozionale } \\
\text { «0» dei verbi copulativi be, have }\end{array}$ \\
\hline $\mathrm{TR}_{\text {prop }}{ }^{+}$ & $\begin{array}{l}\text { Fase segnalata dagli strumenti } \\
\text { morfologici }\end{array}$ & \\
\hline Transizione ${ }^{-}$ & $\begin{array}{l}\text { Componente nozionale dei } \\
\text { verbi parere, fare, dare, }\end{array}$ & $\begin{array}{l}\text { Componente nozionale dei verbi } \\
\text { seem, look, make, become e dei }\end{array}$ \\
\hline
\end{tabular}




\begin{tabular}{|l|lll|l|}
\hline & $\begin{array}{l}\text { diventare, e dei verbi } \\
\text { fraseologici, significato di } \\
\text { cambiamento e di fase dei } \\
\text { verbi derivati }\end{array}$ & \\
\hline Transizione $^{0}$ & $\begin{array}{l}\text { Componente nozionale del } \\
\text { verbo pieno }\end{array}$ & $\begin{array}{l}\text { Componente nozionale del verbo } \\
\text { pieno }\end{array}$ \\
\hline Rema & $\begin{array}{l}\text { Componente nozionale del } \\
\text { verbo pieno }\end{array}$ & $\begin{array}{l}\text { Componente nozionale del verbo } \\
\text { pieno }\end{array}$ \\
\hline
\end{tabular}

Come dimostra la tabella, le differenze tra l'italiano e l'inglese si possono evidenziare nei casi in cui, in italiano, per esprimere la fase dell'azione, esiste la possibilità di adoperare uno strumento morfologico, cioè un tempo grammaticale del verbo nel predicato sintetico, mentre in inglese, per esprimerla, l'unica possibilità è l'uso di un verbo fraseologico nel predicato analitico. Una simile differenza è osservabile nei casi in cui, in italiano, il significato del cambiamento viene riportato con un verbo derivato, mentre in inglese questo significato viene espresso separatamente con verbi come become o turn ecc. La tendenza della lingua inglese all'uso dei predicati analitici si riflette nell'atto di separazione dell'ETM di $\mathrm{TR}_{\text {prop }}$ dalla componente nozionale di transizione o di rema riportata da un elemento non verbale. La separazione di questi due elementi, da una parte, ha come risultato un indebolimento della componente nozionale del verbo predicativo, dall'altra parte mette in rilievo l'elemento non verbale.

Osservando la struttura del predicato si è cercato di evidenziare, a livello sintattico, le manifestazioni della tendenza alla sinteticità della lingua italiana, messe a confronto con le manifestazioni della tendenza all'analiticità della lingua inglese. Un altro scopo è stato quello di evidenziare l'impatto di tali manifestazioni sulla distribuzione di DC. A questo proposito siamo arrivati alla seguente conclusione: Il verbo italiano di un predicato sintetico, grazie alle caratteristiche morfologiche e grazie alle possibilità di derivazione, è in grado di esprimere, oltre alla componente nozionale, anche diverse modificazioni aspettuali, fra le quali il cambiamento di stato. In inglese, al predicato sintetico italiano corrisponde spesso un verbo categoriale con un elemento non verbale, uniti in un predicato analitico. Nel caso del genere, il grado di DC della componente nozionale del verbo italiano supera quello della componente nozionale del verbo inglese. Si può quindi constatare che le differenze morfosintattiche e quelle lessicali tra le lingue in questione si riflettono a livello comunicativo, cioè al livello della PFE.

\section{BIBLIOGRAFIA}

Alisova Tatiana (1972), Strutture semantiche e sintattiche della proposizione semplice in italiano, Firenze, Sansoni.

BALly Charles (1963), Linguistica generale e linguistica francese, Milano, Il Saggiatore.

BAUER Jaroslav, GREPL Miroslav (1975²), Skladba češtiny, Praha, SPN.

BENVENISTE Emile (1994), Problemi di linguistica generale, Milano, Il Saggiatore.

BertinetTo Pier Marco (1991), Il verbo, in Renzi, Salvi 1991, p. 13-161. 
DANEŠ František (1964), A Three-Level Approach to Syntax, TLP 1, Prague, p. 225-240.

DANEŠ František, HLAVSA Zdeněk, GREPL Miroslav (a cura di) (1987), Mluvnice češtiny 3. Skladba, Praha.

DUŠKOVÁ Libuše (1988), Mluvnice současné angličtiny na pozadí češtiny, Praha.

FIRBAS Jan (1959), Thoughts on the communicative function of the English verb, Brno Studies in English, 1, p. 39-68.

FIRBAS Jan (1961), On the Communicative Value of the Modern English Finite Verb, Brno Studies in English, 3, p. 79-98.

FIRBAS Jan (1966), Non-thematic subjects in contemporary English, TLP, 2, Prague, p. 239-256.

FIRBAS Jan (1979), A functional view of 'ordo naturalis', Brno Studies in English, 13 , p. 29-59.

FIRBAS Jan (1991), Il funzionamento del dinamismo comunicativo nella prospettiva funzionale della frase, in Sornicola, Svoboda 1991, p. 194-209.

FIRBAS Jan (1992), Functional sentence perspective in written and spoken communication. Cambridge, Cambridge University Press.

GrePl Miroslav, KARLÍK Petr (1998), Skladba češtiny, Olomouc, Votobia.

HAMPLOVÁ Sylva (1994), K problematice vidovosti v italštině, Praha, UK.

KLÍMOVÁ Eva (2003), Italské určité sloveso z pohledu funkční větné perspektivy ve srovnání s určitým slovesem anglickým. Opava, FPF SU.

KLÍMOVÁ Eva (2004), Il verbo italiano e il verbo inglese sulle scale semantiche, Linguistica Pragensia, XIV/1, p. 32-41.

RENZI Lorenzo, SALVI Giampaolo (a cura di) (1991), Grande grammatica italiana di consultazione, vol. II, Bologna, Mulino.

SORNICOLA Rosanna (1983), Un metodo di analisi della struttura informativa e sue applicazioni all'italiano, SLI, Atti del 17 Congresso Internazionale della Società di Linguistica italiana, p. 3-18.

Sornicola Rosanna, Svoboda Aleš (a cura di) (1991), Il campo di tensione, Napoli, Liguori.

SvoBODA Aleš (1989), Kapitoly z funkční syntaxe, Praha, SPN.

SvobodA Aleš (1991), Le posizioni nell'ordine delle parole ceco dal punto di vista dell'articolazione attuale, in Sornicola, Svoboda 1991, p. 423-452.

VACHEK Josef (1980), Linguistic Characterology of Modern English, Brno.

\section{Fonti degli esempi:}

JOYCE James (1992), Dubliners, London, Penguin Books.

JoYCE James (1995), Gente di Dublino (traduzione di Marina Emo Capodilista), Luigi Reverdito Editore.

MoraVIA Alberto (1992), Gli indifferenti, Milano, Tascabili Bompiani.

MORAVIA Alberto (1975), The Time of Indifference (traduzione di Angus Davidson), Frogmore, Panther.

TOMASI DI LAMPEDUSA Giuseppe (19937 $)$, Il Gattopardo, Milano, Feltrinelli.

TOMASI DI LAMPEDUSA Giuseppe (1996), The Leopard (traduzione di Archibald Colquhoun), London, The Harvill Press. 


\begin{abstract}
The aim of the article is to observe the Italian and the English finite verb form from the functional sentence perspective (FSP) point of view, more precisely to observe the degree of communicative dynamism (CD) of the finite verb form in dependence on the structure of the predicate. In FSP the finite verb form represents several communicative units. One is constituted by its categorial exponents: person, number (PNE), tense, mood (TME). The other is constituted by its notional component. The PNE refers to the Subject and thus participates on the theme. The TME performs the function of transition proper and the notional component of the finite verb form may perform the function of transition or rheme. We follow the notional component of the verbs functioning as copula (Italian essere, avere, parere, sembrare, diventare and English be, have, seem, appear, become, turn), the phase verbs (the Italian cominciare, continuare, smettere and the English begin, continue, stop), and the notional component of full verbs. Thus the function of the notional component of the verb within an analytical predicate is distinguished from that of the verb within a synthetic predicate. The notional component of the Italian derived verbs in the synthetic predicate (Impallidi.) carries higher degree of CD than the notional component of the English verbs of the analytical predicate (She turned pale) the former performing the function of rheme, the later the function of transition.
\end{abstract}

